\title{
ICD and DSM: neuroplasticity and staging are still missing
}

\author{
Stefano Pallanti*
}

Dipartimento di Neuroscienze, Psicologia, Area del Farmaco e Salute del Bambino (NEUROFARBA), University of Florence, Florence, Italy; Department of Psychiatry and Behavioral Sciences, Albert Einstein College of Medicine, New York, New York, USA

The two main diagnostic systems, the International Classification of Diseases (ICD) and the Diagnostic and Statistical Manual of Mental Disorders (DSM), have undergone a number of revisions since their first editions: whereas the fifth edition of the DSM has been published in 2013, the eleventh revision of the ICD is expected by 2018. Although the process of harmonization between the 2 systems is still a debated topic, the forthcoming revision of the ICD is seemingly converging toward the DSM approach in regard to the reclassification of a number of disorders. Nevertheless, the 2 systems still exhibit considerable differences, partly due to their different purposes, development and revision processes, and target audiences. Furthermore, while alternative and innovative classification approaches are emerging with the aim of integrating the latest findings from neuroscience and genomics, both the DSM and ICD still fail to incorporate core concepts such as the clinical staging of psychiatric disorders and "neuroprogression," as well as an adequate consideration of endophenotypes.

Received 9 February 2016; Accepted 9 February 2016; First published online 14 July 2016

Key words: Classification, DSM, ICD, nosology.

\section{Introduction}

The 2 current main diagnostic systems are the International Classification of Diseases (ICD) and the Diagnostic and Statistical Manual of Mental Disorders (DSM). Whereas the ICD covers the entire range of human disease, the DSM focuses on mental disorders only. Both systems have undergone a number of revisions since their first editions: the American Psychiatric Association (APA) released the fifth edition of the DSM in 2013, whereas the World Health Organization (WHO) eleventh revision of the ICD is expected by 2018. The forthcoming revision of the ICD has drawn attention to the similarities and differences between the 2 systems in the classification of mental disorders, especially given the increasing popularity gained by a new classification framework, that is, the Research Domain Criteria (RDoC) project by the National Institute of Mental Health (NIMH).

The ICD and the DSM have different purposes, and this partly accounts for their different foci in the

* Address for correspondence: Stefano Pallanti, Dipartimento di Neuroscienze, Psicologia, Area del Farmaco e Salute del Bambino (NEUROFARBA), University of Florence, Via delle Gore 2H, 50134 Firenze, Italy.

(Email: stefanopallanti@yahoo.it) classification of mental disorders. The DSM is primarily intended for clinical purposes and was developed for mental health professionals; it has a tendency to subtype disorders by developing specifiers. The ICD represents a diagnostic tool for mortality and morbidity statistics, and it is increasingly used to define diseases and study disease patterns, as well as to manage healthcare, monitor outcomes, and allocate resources; therefore, its primary users include physicians, nurses, health workers, researchers, health information managers, policy makers, insurers, and national health program managers. ${ }^{1}$ Conversely, the recent $\mathrm{RDoC}$ project represents an attempt to overcome the limitations of current systems; it focuses on the neurobiological mechanisms and dimensions underlying mental disorders, in order to reach a deeper understanding of the circuitries and networks of psychiatric disorders considered to be responsible for brain diseases. ${ }^{2}$

However, a relevant issue that pertains to both diagnostic systems is the concept of staging and "neuroprogression." Neither the DSM Fifth Edition (DSM-5) nor the draft of the Eleventh Revision of the ICD (ICD-11) has incorporated long-debated concept of clinical staging, which may differentiate early, milder clinical phenomena from those that accompany illness progression and chronicity. This is already a reality in 
several fields of medicine, and is grounded on the assumption that early intervention can produce better clinical and functional outcomes. Nevertheless, it is a currently neglected dimension in most psychiatric disorders $^{3}$ : the concept of clinical staging is emerging in the research on schizophrenia, ${ }^{4}$ together with the aim of an identification of the trajectory of the disorder and the investigation of the after-effects of the duration of untreated disorder, while this seems to be still backward in OCD research.

The idea of staging is, instead, becoming a core concept in explanatory models of illness: the recognition of pleiotropy (that is, the ability of a gene to affect multiple traits) suggests that many disorders may result from shared underlying genetics and different epigenetic events, depending on the timing of stressful/traumatic events.

What is currently missing in both diagnostic systems is the integration of a "clinimetric" perspective,, 5 a concept referring to a number of clinical issues that do not find room in the current taxonomy, comprising clinical staging and severity. Such integration would also inform therapeutics of disorders and may help developing sequential treatment strategies, which have been, up to now, scarcely investigated. A diagnostic system allowing the identification of clinimetric properties would facilitate research and the development of specific treatments strategies in complex clinical pictures and comorbid conditions. ${ }^{7}$

Endophenotypes represent a still debated concept, yet they are a hot topic in neuropsychiatric research. They can be conceptualized as a special kind of biomarker, encompassing heritable neurobiological and neurobehavioral characteristics that play an important role for bridging the gap between the microscopic level (eg, molecular genetics) and the macroscopic level (eg, clinical symptoms) of neuropsychiatric disorders. ${ }^{8,9}$ Neither of the existing nosological systems has so far included an adequate integration of the current knowledge about neural circuits, neurotransmitters and, behavior, as proposed by the National Institute of Mental Health (NIMH) Research Domain Criteria (RDoC) projec. ${ }^{10}$

In the Research Agenda for DSM-5 (Charney et al., 2002 in: Kupfer et al., 2002) ${ }^{11}$, the need to translate basic and clinical neuroscience research findings into a new classification system for all psychiatric disorders based on pathophysiologic and etiological processes was emphasized. In this perspective, the identification of specific endophenotypes would help in both the diagnostic and therapeutic processes, by the means of a deeper understanding of the pathophysiologic processes specific to a disorder and the identification of differential responses to specific treatments, respectively. ${ }^{12}$

This special issue of CNS Spectrums will encompass a number of contributions that examine the differential classification of mental disorders in DSM-5 and ICD, and they will discuss key issues pertaining the revision processes and future directions for research. Specifically, Hollander and Doernberg discuss the diagnostic evolution of neurodevelopmental disorders and examine the differences between the DSM-5 and ICD-10, anticipating and advising the forthcoming ICD-11. This review will also focus on alternative approaches, such as RDoC, and on how the incorporation of new diagnostic directions could have a great effect on treatment development.

Anxiety disorders will be reviewed by Bandelow, and specifically, separation anxiety disorder as a new diagnosis in DSM-5 will be discussed by Baldwin and colleagues, who will also examine its relationship with other anxiety disorders. Previously included within the disorders usually first diagnosed in infancy, childhood, or adolescence, separation anxiety disorder could be diagnosed in adults only retrospectively. Recently, the disorder revealed an unexpectedly high prevalence in adults, often in individuals with an onset of symptoms after the teenage years, and this explains its re-classification in DSM-5.

Posttraumatic stress disorder (PTSD) will be examined by Zohar, who addresses the concept of the "window of opportunity" as the time that elapses between the traumatic event and the manifestation of the disorder, in which critical processes take place and relevant interventions may be administrated.

The treatment of depression and the ability of diagnostic systems to define symptoms sufficiently to clearly separate depression from normal mood variations in the general population will be addressed by Montgomery, since both DSM-5 and ICD-10 suffer from a weakness in defining symptoms sufficiently to clearly separate depression from normal mood variations in the general population. The review will also focus on findings, which show a lack of significant separation of active treatment from placebo in mild depression, unlike moderate depression.

Bipolar and related disorders will be discussed by Kasper and colleagues, with a focus on diagnostic criteria changes, although this group of psychiatric disorders is still often difficult to diagnose and has profound negative impact on affected patients.

The debated concept of "behavioral addictions" and the divergent approaches adopted by the 2 diagnostic systems will be addressed by Grant and Chamberlain. Whereas considerable efforts have been made to understand the neurobiological basis of substance addiction, the potentially "addictive" qualities of repetitive behaviors, and whether such behaviors constitute "behavioral addictions," is relatively neglected.

The re-classification of obsessive-compulsive and related disorders (OCRDs) will be discussed by Fineberg and colleagues. The differences and similarities between 
DSM-5 and ICD-10 will be examined, as well as the ICD-11 draft, which seems to be converging toward DSM-5 classification. The review will also focus on affected neurocircuits and the relationship with obsessive-compulsive personality disorder.

The classification of eating disorders will be examined by Erzegovesi and Bellodi, who will focus on the widened diagnoses of anorexia and bulimia nervosa and the new diagnosis of binge eating disorder. In fact, DSM-5 criteria widened the diagnoses of anorexia and bulimia nervosa to less severe forms (and so decreasing the frequency of eating disorders, not otherwise specified (EDNOS) diagnoses), introduced the new category of binge eating disorder, and incorporated several feeding disorders that were first diagnosed in infancy, childhood, or adolescence.

Cosci and Fava will address the clinical inadequacy of the DSM-5 classification of somatic symptoms and related disorders, highlighting 2 major ambiguities: (1) the use of the term "somatic symptoms" reflects an ill-defined concept of somatization and (2) abnormal illness behavior is included in all diagnostic rubrics, but it is never conceptually defined. The authors will present a review of literature, proposing an alternative transdiagnostic model.

Psychotic disorders and the re-organization of this chapter in the DSM-5 will be discussed by Fleischhacker and Biedermann: DSM-5 has eliminated schizophrenia subtypes and replaced them by a dimensional approach based on symptom assessments. ICD-11 will most likely go in a similar direction, as both manuals are planned to be more harmonized, although some differences will remain in details and the conceptual orientation. Next to these modifications, ICD-11 will provide a transsectional diagnostic criterion for schizoaffective disorders and a reorganization of acute and transient psychotic and delusional disorders.

Catatonia will be addressed separately, consistent with the DSM-5 classification, by Strik, with a focus on the neuroimaging of this condition. Neuroimaging reports pointed to reduced resting state activity and reduced task activation in motor areas of the frontal and parietal cortex, whereas suspected neurotransmitter systems include GABA and glutamate. The new classification of catatonia will foster more clinical research and neuroscientific approaches by testing catatonia in various populations applying stringent criteria.
Finally, gender and cultural issues in psychiatric nosological classification systems will be addressed by Suliman and colleagues. They will highlight the tension between a gender- and culture-free nosology (solely biological) and a contextually relevant understanding of mental illness.

\section{Disclosures}

Stefano Pallanti has nothing to disclose.

\section{REFERENCES:}

1. World Health Organization. International Classification of Diseases (ICD) Information Sheet. http://www.who.int/classifications/icd/ factsheet/en/. Accessed April 2, 2016.

2. Insel TR. The NIMH Research Domain Criteria (RDoC) project: precision medicine for psychiatry. Am J Psychiatry. 2014; 171(4): 395-397.

3. Fava GA, Kellner R. Staging: a neglected dimension in psychiatric classification. Acta Psychiatr Scand. 1993; 87(4): 225-230.

4. McGorry PD, Hickie IB, Yung AR, et al. Clinical staging of psychiatric disorders: a heuristic framework for choosing earlier, safer and more effective interventions. Aust N Z J Psychiatry. 2006; 40(8): 616-622.

5. Feinstein AR. T. Duckett Jones Memorial Lecture: the Jones criteria and the challenges of clinimetrics. Circulation. 1982; 66(1): 1-5.

6. Fava GA, Rafanelli C, Tomba E. The clinical process in psychiatry: a clinimetric approach. J Clin Psychiatry. 2012; 73(2): 177-184.

7. Pallanti S, Grassi G. Pharmacologic treatment of obsessivecompulsive disorder comorbidity. Exp Opin Pharmacother. 2014; 15(17): 2543-2552.

8. Gottesman II, Gould TD. The endophenotype concept in psychiatry: etymology and strategic intentions. Am J Psychiatry. 2003; 160(4): 636-645.

9. Insel TR, Cuthbert BN. Endophenotypes: bridging genomic complexity and disorder heterogeneity. Biol Psychiatry. 2009; 66(11): 988-989.

10. Insel T, Cuthbert B, Garvey M, et al. Research domain criteria (RDoC): toward a new classification framework for research on mental disorders. Am J Psychiatry. 2010; 167(7): 748-751.

11. Charney DS, Barlow DH, Botteron K, Cohen JD, Goldman D, Gur RE, Lin KM, López JF, Meador-Woodruff JH, Moldin SO, Nestler EJ, Watson SJ, Zalcman SJ. Neuroscience research agenda to guide development of a pathophysiologically based classification system. In: Kupfer, David J. (Ed); First, Michael B. (Ed); Regier, Darrel A. (Ed), (2002). A research agenda for DSM-V., (pp. 31-83). Arlington, VA, US: American Psychiatric Association, xxiii, 307 pp.

12. Phillips ML. The emerging role of neuroimaging in psychiatry: characterizing treatment-relevant endophenotypes. $\mathrm{Am} \mathrm{J}$ Psychiatry. 2007; 164(5): 697-699. 